\title{
Facility Power Usage Modeling and Short Term Prediction with Artificial Neural Networks
}

\author{
Sunny Wan and Xiao-Hua Yu \\ Department of Electrical Engineering, California Polytechnic State University, \\ San Luis Obispo, CA 93407, USA
}

\begin{abstract}
Residential and commercial buildings accounted for about 68\% of the total U.S. electricity consumption in 2002. Improving the energy efficiency of buildings can save energy, reduce cost, and protect the global environment. In this research, artificial neural network is employed to model and predict the facility power usage of campus buildings. The prediction is based on the building power usage history and weather conditions such as temperature, humidity, wind speed, etc. Different neural network configurations are discussed; satisfactory computer simulation results are obtained and presented.
\end{abstract}

Keywords: Power prediction, building energy management, artificial neural network applications.

\section{Introduction}

With the limited resources of fossil fuel and the ever-increasing energy demand, the studies on energy management have become more and more important. It is reported that residential and commercial buildings accounted for about $68 \%$ of the total U.S. electricity consumption in 2002 [1]. An efficient energy management system of a building can optimize energy use, improve comfort, reduce building operational cost and energy related emissions, and thus protect the global environment.

Facility management is essential for building maintenance and functioning. The energy consumption related with facility operations includes, but not limited to, HVAC (Heating, Ventilating, and Air Conditioning), lighting, water supply, etc.

Facility power usage modeling and short term prediction plays an important role in an adaptive energy management system. Based on the model and/or prediction, a control signal can be generated on-line to minimize the power consumption and thus optimize the system performance. In addition, it can also be used as an integral part of the building SHM (Structural Health Monitoring) system, to detect and identify possible equipment failure, and to provide information for the retrofit of existing buildings.

Power usage prediction is often based on statistics or the numerical modeling of historical data, such as linear regression models [10]. However, these models are 
usually not accurate and the order of linear regression model can be very high. In addition, recent studies show that building power usage is also related with other factors such as the building occupancy and weather conditions.

The LCEA (Life Cycle Energy Assessment) of buildings is a systematic and comprehensive way to assess building energy consumption. It is part of the ISO 14,000 series of standards on environmental management [2]. LCEA is based on the model of the physical structure of the building, such as the materials used in building envelope (i.e., the separation between the interior and the exterior environments of a building - for example, the brick wall), the gross volume and floor area of the building, floor finish, ceiling height, etc. LCEA provides a general guideline to estimate building energy consumption; however, it lacks the precision and details when it is used for short-term energy management.

There have been some developments in recent years in the application of artificial neural networks (ANN) for building power usage modeling and prediction, such as [3], [4], and [5]. It is well known that an artificial neural network with appropriate size can be employed to approximate any measurable function once it is fully trained ([7], [9]). The approach based on neural network has some significant advantages over conventional methods, such as adaptive learning and nonlinear mapping.

An energy forecasting model of a building based on artificial neural network is discussed in [3]. The building is modeled and calibrated using a software package ("DOE-2.1E Building Energy Analysis Program") first; then neural network is trained using the simulated data generated by the software. It is recommended that future neural network modeling should be developed and tested using "real", or "actual" building measurement data.

In [4], the relationship between the energy consumption and the work shift of different units in a dairy firm is studied. The inputs to the neural network include the specific hours in a day for different types of activities (i.e., working, not working, and washing) at various processing units. However, the impact of weather conditions is not considered.

The prediction of thermal energy consumption of a hospital using neural networks is investigated in [5]. The time-series prediction is based on the measurements that are recorded by meters, such as the natural gas consumption, the cold and hot water consumption, the external temperature, as well as the temperature inside the building. Note this is still a rough estimation due to the low data sampling rate (about two hours). In addition, temperature is the only weather related parameter that is included in the neural network model.

This paper focuses on the modeling and short-term prediction of facility power usage for buildings at Cal Poly (California Polytechnic State University) SLO (San Luis Obispo) campus. The past power consumption data is provided by the Dept. of Engineering and Utilities Facility Services at Cal Poly; and the data on the her of San Luis Obispo County is obtained from internet [6]. Simulation results show that artificial neural network can successfully model and predict facility power usage for campus buildings. 


\section{The Neural Network Model for Power Consumption Prediction}

In this section, the neural network model for building power usage modeling and prediction is discussed. The building power usage can be modeled as a nonlinear function of several parameters that include the history of power usage as well as the current weather conditions:

$$
P(n+1)=f[P(n), T(n), W(n), \cdots] .
$$

where $n$ is the time index; $P(n+1)$ is the prediction and $P(n)$ is the current building power usage; $T(n), W(n)$ etc. are parameters that are related with the current weather condition such as temperature, wind speed, etc.

A multi-layer feedforward neural network model is proposed for this application. It has an input layer, an output layer, and one or more hidden layer(s). As indicated in Eq. (1), the neural network inputs include $P(n)$ and the measurements of weather condition while its output is the prediction $P(n+1)$. That is, the neural network model is a multi-input, single-output system. Fig. 1 shows the neural network configuration when one hidden layer is employed:

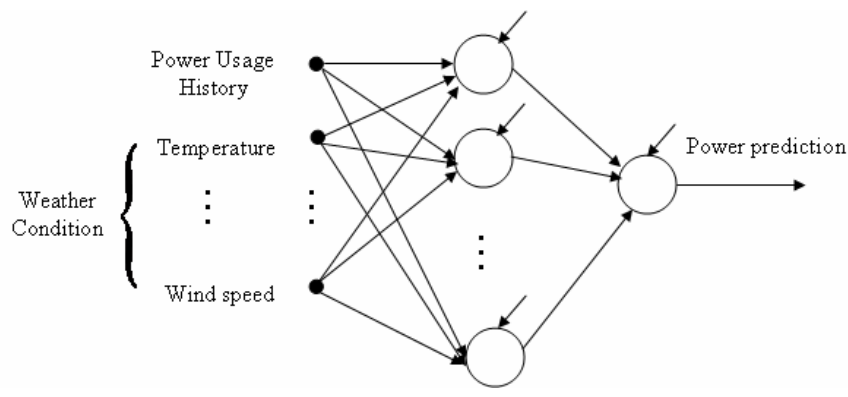

Fig. 1. The Neural network model for power usage prediction

The activation function for each hidden neuron is chosen as the sigmoid function:

$$
f(x)=\frac{1}{1+e^{-x}} .
$$

The activation function for the neuron in the output layer is simply a linear function.

The weights of the neural network are initialized at random, and then updated to minimize the following objective function:

$$
[e(n)]^{2}=\frac{1}{2}[\hat{P}(n)-P(n)]^{2}=\frac{1}{2}\left[y_{N N}(n)-y_{d}(n)\right]^{2} .
$$

where $\hat{P}(n)$ is the prediction (i.e., the NN output $\left.y_{N N}(n)\right)$ and $P(n)$ is the actual building power usage (i.e., the desired output $\left.y_{d}(n)\right)$. 
The Levenberg-Marquardt algorithm is employed to train the neural network:

$$
W(k+1)=W(k)+\Delta W .
$$

and:

$$
\Delta W=\left(J_{a}{ }^{T} J_{a}+\mu I\right)^{-1} J_{a}{ }^{T} e .
$$

where $J_{a}$ is the first order derivative of the error function with respect to the neural network weight (also called the Jacobian matrix); $e$ is the output error (i.e., the difference between the neural network output and the desired output); $\mu$ is a learning parameter, and $k$ is the index of iterations.

Before training the neural network model, the number of hidden layers and the number of hidden neurons in each layer must be specified. As we know, the dimension (or size) of a neural network may have a great impact on neural network learning. In general, a larger neural network (with more hidden layers and/or more hidden neurons) is able to approximate more complex nonlinear functions; however it may require more memory space and longer computation time. Besides, its generalization ability may be poor and thus causes the "over-fitting" problem (that is, the neural network can fit well through the training data while fail to generate the correct output for testing data). On the other hand, a smaller neural network runs faster but may yield a higher training error (called the "under-fitting" problem). Therefore, choosing the appropriate network size becomes a critical issue in the design of artificial neural networks [8]. This issue will be further discussed in the next section.

\section{Simulation Results}

In this section, the proposed neural network model is applied to predict the facility power usage of a building on Cal Poly campus.

Most building energy management applications use monthly data [10]. The Dept. of Engineering and Utilities Facility Services at Cal Poly provides us the power consumption data of building 1 (Administration Building) in November 2008. The data sampling period is 15 minutes, resulting totally 2878 measurements. The weather condition of the San Luis Obispo County is obtained from the web ([5]), which includes temperature $(\mathrm{F})$, dew point $(\mathrm{F})$, pressure (inch), wind speed (mph), wind gust (mph), humidity (\%), and rainfall rate (hourly). Note that this data set is not sampled uniformly - i.e., the time interval between two adjacent measurements is not a constant. For example, some of the measurements are 5 minutes apart; while others may be 6 minutes, 10 minutes, or even up to 15 minutes apart. To ensure the consistency of data, the nearest-neighbor interpolation (also known as proximal interpolation), a piecewise-constant interpolation algorithm, is employed to "resample" the data every 15 minutes. At each sampling instant, if the original measurement data is not available, this algorithm then sets the value of data at this 
point to be the same as the value of the immediate previous measurement (i.e., the measurement that was taken right before the current sampling time instant).

The entire database contains 2,878 sets (or points). Among them, 2,000 points are used for neural network training and the rest 878 points are used for testing. The power consumption of the building varies from $54 \mathrm{~kW}$ to $215 \mathrm{~kW}$, with an average of $101.552 \mathrm{~kW}$, a median of $80 \mathrm{~kW}$, and a mode of $68 \mathrm{~kW}$ (in statistics, the term "mode" refers to the value that occurs the most frequently in a data set or a probability distribution).

To determine the appropriate size of neural network for this application, two neural networks with different architectures are employed in the simulation, and their performances are compared. Both networks have 8 input neurons ( 7 for the measurements on weather and 1 for the current power usage) in the input layer; and 1 output neuron in the output layer (for power prediction). Network 1 has one hidden layer with 9 neurons while network 2 has two hidden layers, with 10 neurons in the first hidden layer and 5 neurons in the second hidden layer.

The computer simulation results of the proposed neural network model for building power usage are shown in Fig. 2 - Fig. 5. On-line training is employed in this research. Fig. 2 shows the performance of network 1 in training phase, where the solid line represents the desired output and the dotted line represents the output of neural network. Similarly, Fig. 3 illustrates the training results of network 2. The neural network performance in testing phase is shown in Fig. 3 (for network 1) and Fig. 4 (for network 2), where the solid line represents the actual building power consumption and the dotted line represents the neural network prediction.

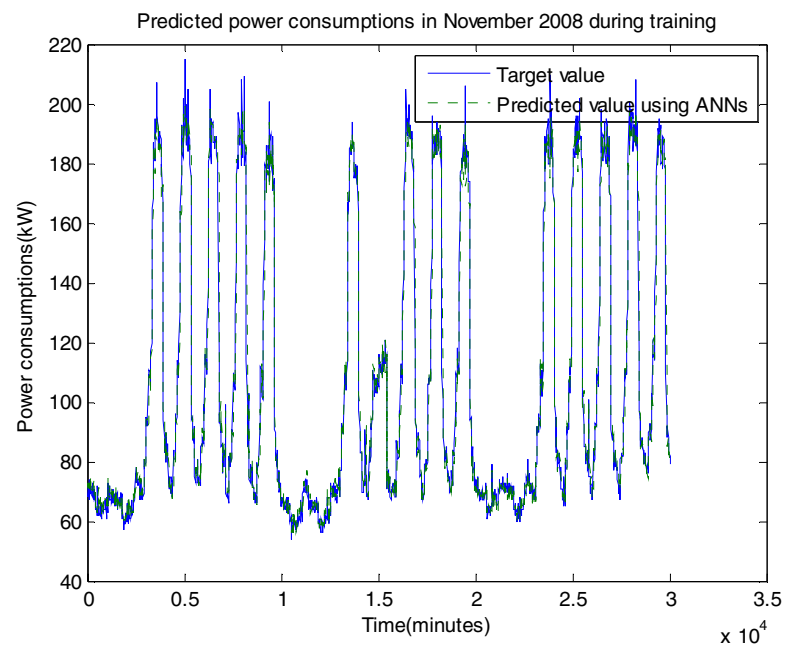

Fig. 2. Neural Network 1 Training Results 


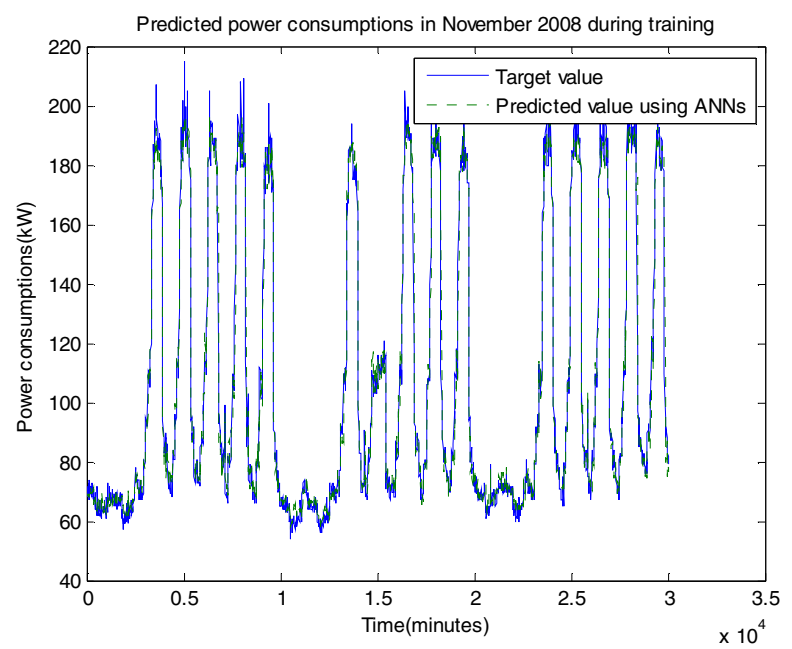

Fig. 3. Neural Network 2 Training Results

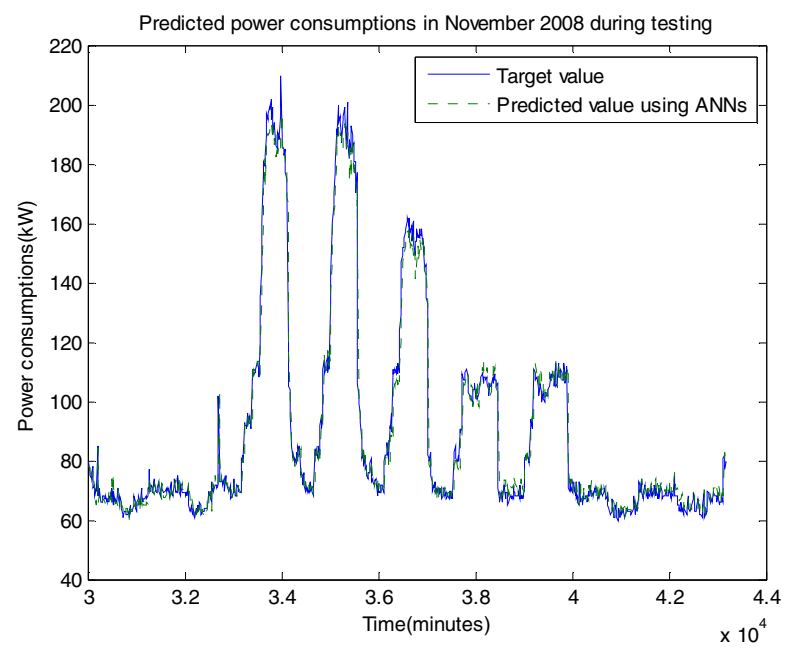

Fig. 4. Neural Network 1 Testing Results

Both neural networks can successfully predict the building power consumption based on weather conditions and the power usage history. Further analysis on the training error and the testing error is given in Table 1, where " 1 " is for networks 1 , " 2 " is for network 2, " $R$ " is the training phase, " $E$ " is the testing phase, "Std. Dev." is the standard deviation of the error, and "Avg." is the average, "Max." is the maximum, "RMS" is the root-mean-square value of errors. The statistics values shown in the tables indicate that the output error of network 1 is unbiased with a smaller standard deviation, average, maximum, median, mode, and RMS value, in both training and testing phases. For example, the RMS value of testing error is 
$6.8766 \mathrm{~kW}$ for network 1 (about $5.66 \%$ of the average power consumption of the building) while the RMS value of testing error is $10.9224 \mathrm{~kW}$ for network 1 (about $10.76 \%$ of the average power consumption of the building). Therefore, we conclude that network 1 yields better overall performance than network 2 .

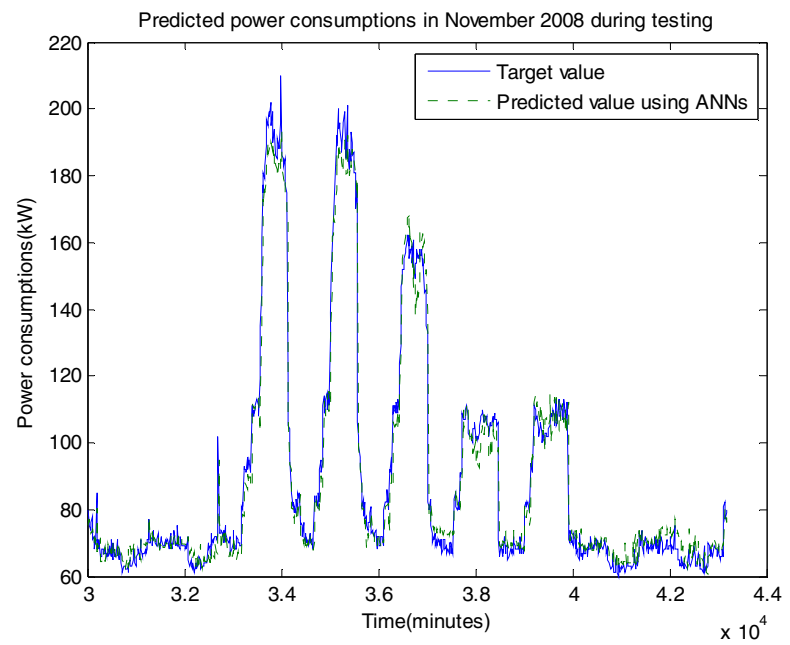

Fig. 5. Neural Network 2 Testing Results

Table 1. Statistics of Neural Network Performance (Standard Deviation, Average, Maximum, Median of Error, Mode, and RMS value of Error)

\begin{tabular}{|c|c|c|c|c|c|c|c|}
\hline NN & Phase & Std. Dev. & Avg. & Max. & Median & Mode & RMS \\
\hline 1 & R & 6.8783 & -0.0006 & 25.2227 & -0.1103 & -1.6127 & 6.8766 \\
\hline 1 & E & 5.7397 & 0.2785 & 30.0599 & -0.0428 & -2.3454 & 5.7432 \\
\hline 2 & R & 6.9312 & 0.0282 & 25.8888 & -0.1814 & -2.8192 & 12.7105 \\
\hline 2 & E & 6.4726 & 0.3032 & 30.8219 & -0.2951 & -4.4821 & 10.9224 \\
\hline
\end{tabular}

\section{Conclusions}

An approach to model and predict building energy consumption based on artificial neural network is studied in this paper. Satisfactory computer simulation results are obtained and presented. More tests will be conducted to further investigate the performance of the neural network model in the future. For example, this approach can be applied to and tested on the data collected from different buildings and/or different times of the year. Also, the raw measurement data may contain noise and outliers. Pre-processing the raw data using adaptive filtering and/or outlier detection may speed up neural network learning and improve the neural network generalization ability. 
Acknowledgments. The authors would like to thank Mr. D. Elliot for providing the data that is used in this research.

\section{References}

1. U.S. Environmental Protection Agency Green Building Workgroup, Buildings and the environment: a statistical summary, http://www. epa.gov/greenbuilding/pubs/gbstats.pdf

2. Kofoworola, O., Gheewala, S.: Life cycle energy assessment of a typical office building in Thailand. Energy and Buildings 41, 1076-1083 (2009)

3. Cohen, D., Krarti, M.: A neural network modeling approach applied to energy conservation retrofits. In: Proceedings of the Fourth International Conference on Building Simulation, pp. 423-430 (1995)

4. Frosini, L., Petrecca, G.: System identification for the prediction of the electric energy consumption of a dairy firm. In: Proceedings of the 2001 IEEE Mountain Workshop on Soft Computing in Industrial Applications (2001)

5. Frosini, L., Petrecca, G.: Neural networks for energy flows prediction in facility systems. In: Proceedings of 1999 IEEE Midnight-Sun Workshop on Soft Computing Methods in Industrial Applications (1999)

6. Weather Underground, http: / / www . wunderground. com/weatherstation/index.asp

7. Haykin, S.: Neural Networks: A Comprehensive Foundation, 2nd edn. Prentice Hall, Englewood Cliffs (1999)

8. Geman, S., Bienenstock, E., Doursat, R.: Neural networks and the bias/variance dilemma. Neural Computation 4, 1-58 (1992)

9. Sjoberg, J., Hjalmerso, H., Ljung, L.: Neural networks in system identification. In: Proceedings of the 10th IFAC symposium on system identification (1994)

10. Begg, C.: Energy: management, supply and conservation. Butterworth-Heinemann (2002) 